\title{
A Hybrid Boundary Element Unstructured Transmission-line (BEUT) Method for Accurate 2D Electromagnetic Simulation
}

\author{
Daniel Simmons, Kristof Cools, and Phillip Sewell
}

\begin{abstract}
Time domain electromagnetic simulation tools have the ability to model transient, wide-band applications, and non-linear problems. The Boundary Element Method (BEM) and the Transmission Line Modeling (TLM) method are both well established numerical techniques for simulating time-varying electromagnetic fields. The former surface based method can accurately describe outwardly radiating fields from piecewise uniform objects and efficiently deals with large domains filled with homogeneous media. The latter volume based method can describe inhomogeneous and non-linear media and has been proven to be unconditionally stable. Furthermore, the Unstructured TLM
\end{abstract} (UTLM) enables modeling of geometrically complex objects by using triangular meshes which removes staircasing and unnecessary extensions of the simulation domain. The hybridization of BEM and UTLM which is described in this paper is named the Boundary Element Unstructured Transmission-line (BEUT) method. It incorporates the advantages of both methods. The theory and derivation of the 2D BEUT method is described in this paper, along with any relevant implementation details. The method is corroborated by studying its correctness and efficiency compared to the traditional UTLM method when applied to complex problems such as the transmission through a

D. Simmons, K. Cools, and P. Sewell are with the George Green Institute for Electromagnetics Research at the University of Nottingham, Nottingham, NG7 2RD, UK. E-mail: daniel.simmons@nottingham.ac.uk.

Manuscript submitted to Journal Of Computational Physics, January 29, 2016. system of Luneburg lenses and the modelling of antenna radomes for use in wireless communications.

Index Terms-2D; UTLM; BEM; MoM; coupling; scattering.

\section{INTRODUCTION}

The simulation of transient electromagnetic (EM) transmission problems is important for all aspects of design and testing of sensitive electronics and antennas. Time domain numerical solvers have evolved rapidly, on the one hand due to the rapid increase in computational power over the last few decades and on the other hand due to a number of breakthroughs in the efficient solution of linear systems that lie at the heart of the algorithms.

Many advances are constantly being applied to integral equation (surface) techniques and differential equation (volume) techniques. By creating a hybrid solver which can be seamlessly integrated, we can enjoy the advantages of each technique, without modifying the underlying core methods. Specifically, a volume technique can be used to model the fields inside the scatterers and a surface technique can be used to model the external field interactions and radiation conditions.

The Time Domain (TD) Boundary Element Method (BEM) can be solved by a marching-on-in-time technique which gives an accurate description of outwardly 
radiating fields because it uses the explicit expression for the Green function to represent these radiating fields. The method decreases the dimensionality of the problem by 1 dimension which, when combined with modern matrix-vector product acceleration techniques, leads to a method whose computational efficiency is second to none. For details of the state-of-the-art discretization and implementation of marching-on-in-time space-time Galerkin methods, the reader is referred to, for example, [1]-[5].

The Unstructured Transmission-Line Modeling (UTLM) method is a time domain volume technique which is unconditionally stable for all time (subject to a maximum timestep constraint) and can model complex, non-linear materials with complex geometries. Curved surfaces can be represented in the mesh with much higher accuracy than is possible with the Cartesian meshing required of structured TLM, thus avoiding staircasing errors. Unfortunately just like the structured TLM, the UTLM method requires the use of Approximate Boundary Conditions (ABCs) to model the radiating behaviour of the fields at the boundary of the simulation domain. Moreover, for these $\mathrm{ABC}$ s to be accurate, the simulation domain needs to be extended beyond the domain occupied by the device under study, leading to an increase in the size of the problem and thus an increase in solution time [6,7]. Finally, the ability to model plane wave excitations and compute radar cross sections, though possible, is not immediately available using TLM [8].

UTLM can be compared to other time domain volume techniques such as the Finite-Difference Time-Domain (FDTD) and the Finite Element Method (FEM) which are both well-established numerical modelling methods. These methods also have the ability to model inhomogeneous and complex media [9]-[11], but only FEM is naturally constructed for unstructured grids (though
FDTD has been extended to non-orthogonal and unstructured meshes previously [12,13]). FDTD has the advantage of being exceptionally simple to implement and FDTD meshes can be terminated with very good absorbing boundary conditions. However, the exact location of boundaries can be problematic due to the offset nature of the electric and magnetic field grids, and the appealing simplicity is lost when attempting to apply FDTD to unstructured meshes. FEM can naturally handle complex geometries and dispersive materials, and has the ability to model multi-physics applications, however it is more difficult to implement compared to FDTD, and its meshes can become very complex [14]. Unlike UTLM and FDTD, FEM is an implicit time-marching scheme i.e. the solution of a linear system via a matrix inversion is computed at each time step, which if directly solved is computationally expensive. Iterative solvers can be used, which have roughly linear memory and compute requirements, but rely on the appropriate use of dedicated preconditioners. Alternatively, the sparse matrix seen in FEM can be approximated to a diagonal matrix via "mass lumping", but this technique can give an unstable algorithm which depends heavily on the problem [15].

As with UTLM, FDTD and FEM do not include radiation conditions for open regions. This is overcome by hybridizing with an efficient integral equation technique, as suggested in $[16,17]$ for FDTD-BEM solvers, and $[18,19]$ for FEM-BEM solvers. Also, the transmission line description of the low frequency response of the domain automatically guarantees stability during runtime, i.e. the output energy equals the input energy, which is especially useful for large simulations. In FEM stability is regulated by bounds that contain hard to estimate constants and that depend on the spatial meshing and temporal oversampling. Given the sensitivity of the stability of TD-BEM solvers, coupling to the trivially 
stable UTLM is considered to be a more conservative choice. Finally, UTLM lends itself to the relatively easy inclusion of more exotic media such as meta-materials, cells containing wires, and active media [20]-[23].

It must be noted that there are methods to obtain good absorbing boundary conditions through the use of a Perfectly matched Layer (PML) in TLM [24][26], however these methods are still inferior to PMLs previously implemented in FDTD $[27,28]$. The use of BEM to truncate the UTLM mesh gives more accurate boundary conditions that can be applied directly to the surface of the scatterers and also allows spatially distinct scatterers to interact without modelling the space between. However, in order for the UTLM-BEM coupling to become competitive in complexity with a pure UTLM scheme, the convolution central to the BEM part of the algorithm needs to be sped up by time domain matrix-vector accelerators such as the Plane Wave TimeDomain (PWTD) algorithm [29,30] or the Time Domain Adaptive Integral Method (TD-AIM) [31,32].

Fig. 1 shows a typical example of a device comprising complex materials inside spatially distinct and well separated regions. Scattering by and transmission through such a device is most efficiently modelled by a method hybridizing the UTLM and the BEM methods. The advantages of this hybridized scheme are:

- Modeling of complex, non-linear media [33] with geometrically complex features

- Perfect radiating boundary conditions

- Straightforward excitation by plane waves

- Free space region does not need to be meshed, enabling more efficient computation of scattering problems where free space dominates

Previous hybridizations between TLM and BEM have been attempted [34]-[40]. However these solvers either contain complicated connection processes that require

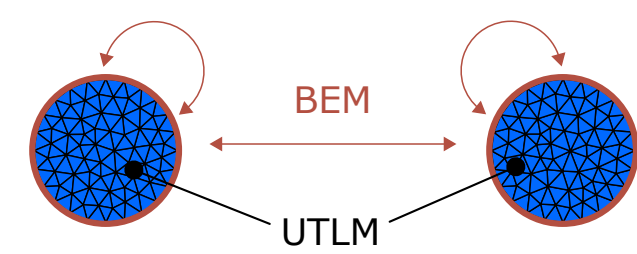

Fig. 1. Arbitrary objects modelled using UTLM, separated by free space modelled by BEM.

a large number of discrete Green's functions, contain discretisation errors on subdomain boundaries (therefore requiring padding between the object and the TLM/BEM interface), or require the use of the inaccurate TLM ABCs. They do not take advantage of unstructured meshes or take into account recent advancements that make BEM and TLM more robust, stable and accurate.

The novel hybrid method described in this paper is called the Boundary Element Unstructured Transmission-line (BEUT) method. It is conceptually very simple and can be easily applied to existing solvers of the two underlying methods. In fact its derivation is directly linked to the construction of the Poggio-MillerChan-Harrington-Wu-Tsai (PMCHWT) integral equation for the modelling of transmission problems through piecewise homogeneous devices [41]. The key ingredient is the construction of a representation formula valid on the (inner) boundary of the TLM governed regions.

This paper is organised as follows: in sections II and III, we give an overview of BEM and UTLM, respectively, and section IV derives the novel BEUT method. Finally, section V demonstrates the technique by simulating the scattering by two spatially distinct Luneburg lenses, and the scattering between a dipole antenna and a radome, along with comparisons of speed and accuracy with purely UTLM simulations. 


\section{INTRODUCTION TO BEM}

To construct the BEM formulas in 2D, we start from the time domain representation formulas. Consider a domain bounded by the contour, $\Gamma$. The exterior unit normal to $\Gamma$ is denoted by $\hat{\boldsymbol{n}}$ and the counter-clockwise directed unit tangential is denoted by $\hat{t}$. For the $2 \mathrm{D}$ Transverse Magnetic (TM) case, the representation formulas can be written as

$$
\left(\begin{array}{c}
e_{z} \\
h_{t}
\end{array}\right)=\left(\begin{array}{cc}
\frac{1}{2}+D & -\frac{\eta}{c} S \\
-\frac{c}{\eta} N & \frac{1}{2}-D^{\prime}
\end{array}\right)\left(\begin{array}{c}
e_{z} \\
h_{t}
\end{array}\right)+\left(\begin{array}{c}
e_{z}^{i} \\
h_{t}^{i}
\end{array}\right)
$$

where $\eta=\sqrt{\mu / \epsilon}$ is the characteristic impedance of the background medium and $c=\sqrt{\mu \epsilon}$ is the wave propagation speed within the background medium. The tangential components of the electric and magnetic fields are represented as $e_{z}$ and $h_{t}\left(=\hat{\boldsymbol{t}} \cdot \boldsymbol{h}_{x y}\right)$ respectively, and the superscript $i$ denotes the incident field.

The operators appearing in eq. (1) are defined as

$$
\begin{aligned}
D \varphi\left(\boldsymbol{r}^{\prime}, t\right) & =\int_{\Gamma} \frac{\partial g}{\partial n^{\prime}}(R, t) * \varphi\left(\boldsymbol{r}^{\prime}, t\right) d r^{\prime} \\
D^{\prime} \varphi\left(\boldsymbol{r}^{\prime}, t\right) & =\int_{\Gamma} \frac{\partial g}{\partial n}(R, t) * \varphi\left(\boldsymbol{r}^{\prime}, t\right) d r^{\prime} \\
S \varphi\left(\boldsymbol{r}^{\prime}, t\right) & =\int_{\Gamma} g(R, t) * \frac{\partial \varphi}{\partial t}\left(\boldsymbol{r}^{\prime}, t\right) d r^{\prime} \\
N \varphi\left(\boldsymbol{r}^{\prime}, t\right) & =-\int_{t} \int_{\Gamma} \frac{\partial^{2} g}{\partial n \partial n^{\prime}}(R, t) * \varphi\left(\boldsymbol{r}^{\prime}, t\right) d r^{\prime} d t
\end{aligned}
$$

where $\varphi$ is an arbitrary vector, $t$ is time, $*$ indicates a temporal convolution, and $R=\left|\boldsymbol{r}-\boldsymbol{r}^{\prime}\right|$.

The 2D time domain Green function is expressed as the field radiated by a Dirac source, represented here as

$$
g(R, t)=\frac{H(t-R / c)}{2 \pi \sqrt{t^{2}-(R / c)^{2}}}
$$

where $H$ denotes the Heaviside function.

To reduce the order of the singularity contained by the hypersingular integrals in $N$, we apply the usual integration by parts manipulation [42] to produce the combination of a singular contribution, $N_{s}$, and a hyper- singular contribution, $N_{h}$, which are defined respectively as

$$
\begin{aligned}
& N_{s} \varphi\left(\boldsymbol{r}^{\prime}, t\right)=\frac{1}{c^{2}} \hat{\boldsymbol{t}} \cdot \int_{\Gamma} \hat{\boldsymbol{t}}^{\prime} \cdot g(R, t) * \frac{\partial \varphi}{\partial t}\left(\boldsymbol{r}^{\prime}, t\right) d r^{\prime} \\
& N_{h} \varphi\left(\boldsymbol{r}^{\prime}, t\right)=-\hat{\boldsymbol{t}} \cdot \int_{\Gamma} \nabla g(R, t) * \int_{t}\left[\nabla^{\prime} \varphi\left(\boldsymbol{r}^{\prime}, t\right)\right] d t d r^{\prime}
\end{aligned}
$$

where the divergence and the gradient reduce to simple derivatives along the boundary in the counter-clockwise direction.

In this contribution, we use a straightforward implementation of BEM and UTLM which have computational complexities of $\mathcal{O}\left(N_{S}^{2} N_{t}^{2}\right)$ and $\mathcal{O}\left(N_{V} N_{t}\right)$ respectively, where $N_{V}$ and $N_{S}$ denote the number of spatial field sampling points within the volume and on the surface of the scatterer respectively, and $N_{t}$ is the number of timesteps. However, the BEM implementation could be further accelerated using techniques such as the Time Domain Adaptive Integral Method (TD-AIM) [31,32] which uses a spatial and temporal Fast Fourier Transform (FFT) for computing convolutions, and the Plane Wave Time-Domain (PWTD) algorithm [29,30] which aggregates far-fields from source sub-scatterers into plane waves which are then superimposed onto the observer.

To discretize the formulations defined in (1), the unknown fields must be expanded as a linear combination of spatial and temporal basis functions. The temporal basis functions must be at least piecewise linear because of the presence of the derivatives in the operators $S$ and $N_{s}$, hence continuous, piecewise linear, nodal time basis functions are used [4]. For the spatial basis functions, piecewise constant functions (or pulses) $f$, and continuous, piecewise linear functions (or hats) $h$ are used. The hats are constructed on the dual mesh of the contour (as shown in fig. 2b) [43]. For testing, the same basis functions are chosen, but pulses and hats are now normalised w.r.t. one over the length of the corresponding boundary edge (instead of one). This iden- 
tification of the testing and sample values will facilitate the coupling with UTLM later on. The equations are evaluated at equidistant times (a.k.a collocation-in-time), and a Galerkin-in-space scheme is used, which stipulates that the spatial testing functions should be the same as the basis functions.

The discretized form of (1) becomes

$$
\left(\begin{array}{l}
\mathrm{e}_{\mathrm{z}} \\
\mathrm{h}_{\mathrm{t}}
\end{array}\right)=\left(\begin{array}{cc}
\frac{\mathbf{G}}{2}+\mathbf{D} & -\frac{\eta}{c} \mathbf{S} \\
-\frac{c}{\eta}(\mathbf{N} \mathbf{s}+\mathbf{N h}) & \frac{\mathbf{G}^{\boldsymbol{\top}}}{2}-\mathbf{D}^{\prime}
\end{array}\right)\left(\begin{array}{l}
\mathrm{e}_{\mathrm{z}} \\
\mathrm{h}_{\mathrm{t}}
\end{array}\right)+\left(\begin{array}{l}
\mathrm{e}_{\mathrm{z}}^{\mathrm{i}} \\
\mathrm{h}_{\mathrm{t}}^{\mathrm{i}}
\end{array}\right)
$$

where superscript ${ }^{\top}$ denotes the transpose, the matrix vector product here in fact implies a discrete convolution, e.g.

$$
\left(\mathbf{D} \mathrm{e}_{\mathbf{z}}\right)^{j}=\sum_{k=0}^{j} \mathbf{D}^{k} \mathrm{e}_{\mathbf{z}}^{j-k}
$$

and where the operators are now defined as

$$
\begin{aligned}
\mathbf{D}_{m, n}^{k}= & \int_{\Gamma} \int_{\Gamma} \frac{f_{m}}{l_{m}}(\boldsymbol{r}) h_{n}\left(\boldsymbol{r}^{\prime}\right) \\
& {\left[\frac{\partial g}{\partial n^{\prime}}(R, t) * T(k \Delta t-t)\right] d r^{\prime} d r } \\
\mathbf{D}_{m, n}^{\prime k}= & \int_{\Gamma} \int_{\Gamma} \frac{h_{m}}{l_{m}}(\boldsymbol{r}) f_{n}\left(\boldsymbol{r}^{\prime}\right) \\
& {\left[\frac{\partial g}{\partial n}(R, t) * T(k \Delta t-t)\right] d r^{\prime} d r } \\
\mathbf{S}_{m, n}^{k}= & \int_{\Gamma} \int_{\Gamma} \frac{f_{m}}{l_{m}}(\boldsymbol{r}) f_{n}\left(\boldsymbol{r}^{\prime}\right) \\
& {\left[g(R, t) * \frac{\partial}{\partial t} T(k \Delta t-t)\right] d r^{\prime} d r } \\
\mathbf{N s}_{m, n}^{k}= & \frac{1}{c^{2}}\left(\hat{\boldsymbol{t}} \cdot \hat{\boldsymbol{t}}^{\prime}\right) \int_{\Gamma} \int_{\Gamma} \frac{h_{m}}{l_{m}}(\boldsymbol{r}) h_{n}\left(\boldsymbol{r}^{\prime}\right) \\
& {\left[g(R, t) * \frac{\partial}{\partial t} T(k \Delta t-t)\right] d r^{\prime} d r } \\
\mathbf{N h}_{m, n}^{k}= & \int_{\Gamma} \int_{\Gamma} \nabla \cdot \frac{h_{m}}{l_{m}}(\boldsymbol{r}) \nabla \cdot h_{n}\left(\boldsymbol{r}^{\prime}\right) \\
& {\left[g(R, t) * \int_{t} T(k \Delta t-t) d t\right] d r^{\prime} d r } \\
\mathbf{G}_{m, n}^{k}= & \delta_{k}^{0} \int_{\Gamma} \frac{f_{m}}{l_{m}}(\boldsymbol{r}) h_{m}(\boldsymbol{r}) d r
\end{aligned}
$$

where $f$ and $h$ denote the square and hat basis functions respectively, $l$ is the edge length, $T$ is the temporal basis function, $m$ and $n$ denote the current testing and basis a)

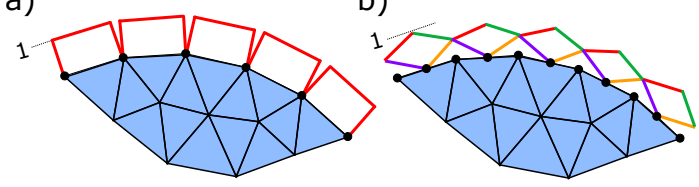

Fig. 2. The boundary section of a scatterer showing a) UTLM basis functions, and b) BEM dual hat basis functions.

functions respectively, $k$ is the current timestep, and the Kronecker delta is defined as

$$
\delta_{j}^{i}= \begin{cases}0 & (i \neq j) \\ 1 & (i=j)\end{cases}
$$

\section{INTRODUCTION TO UTLM}

The TLM method is an established time-domain numerical simulation tool which until 2005 [7] was formulated solely for structured Cartesian meshes. The general TLM algorithm is derived from the established analogy between the local behaviour of EM fields in 3D space and propagation of signals in a suitably interconnected network of 1D transmission lines. The algorithm proceeds by implementing the simple scattering behaviour of signals in a network which models a distinct incremental region of space.

In TLM it is assumed that the electric and magnetic fields are constant over intervals of time $\Delta t$. For each cell, a transmission line circuit is constructed such that:

i) the travel time on each of the constituent transmission lines equals $\Delta t$,

ii) the low frequency response equals that of an equally shaped region of free space,

iii) in the lossless case, only passive or energy conserving elements are present.

In [7], such a circuit is designed for unstructured triangular domains where the Dirichlet-to-Neumann map for a small triangular region can be approximated to first 
order accuracy by a network of transmission lines with the signal transit time along all transmission lines synchronized to the discretization timestep; i.e. a commensurate network is created. It is conventional to describe the circuit in terms of voltages and currents which are related to the electric and magnetic fields at the centers of the mesh edges by

$$
e_{z} \leftrightarrow V \quad h_{t} l \leftrightarrow I
$$

where $l$ is the length of the edge.

A typical UTLM triangle is shown in fig. $3 \mathrm{~b}$. The transmission line circuit comprises two types of line for each port of each triangle: the link line is defined by the perpendicular distance from the port (edge) to the center of the triangle, the stub line is attached to the link line and runs parallel to the edge near the port. To increase the minimum link length and thus increase the minimum timestep (as explained later), the link lengths that cross non-boundary edges are defined as half the distance between connecting triangle centroids, as depicted in Fig. 3c. The link and stub lines are characterised by an impedance $Z_{\text {link }}$, and admittance $Y_{\text {stub }}$, respectively. These parameters depend on the permittivity and permeability of the medium inside the triangle.

In order to match the inductive and capacitive response of the circuit to that of the medium, link and stub admittances need to be chosen as derived in [7],

$$
\begin{aligned}
Y_{\text {link }_{\alpha}} & =\frac{l_{\alpha} \Delta t}{2 \mu \Delta_{\alpha}} \\
Y_{\text {stub }} & =\frac{\epsilon l_{\alpha} \Delta_{\alpha}}{\Delta t}-\frac{l_{\alpha} \Delta t}{2 \mu \Delta_{\alpha}}
\end{aligned}
$$

where the link length associated with port $\alpha$ is given by $\Delta_{\alpha}$. The permeability and permittivity of the medium associated with the triangle are denoted by $\mu$ and $\epsilon$ respectively. To minimize dispersion error and guarantee stability, the stub admittances must all be positive, which a)

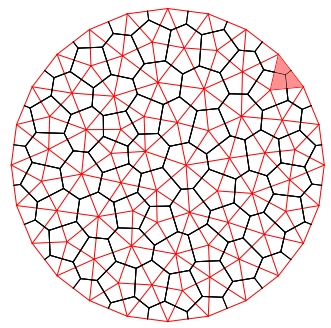

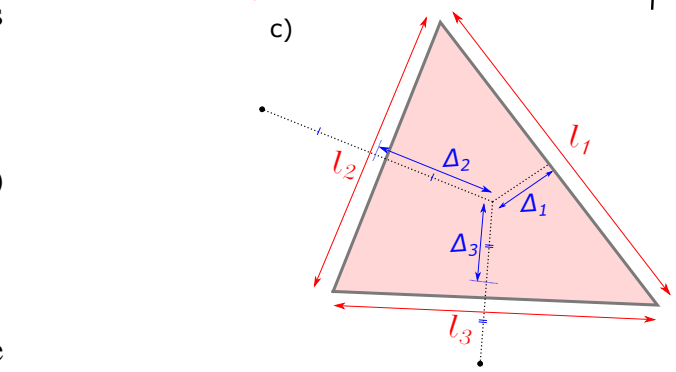

b)

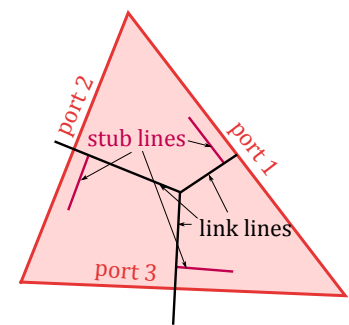

Fig. 3. An example UTLM mesh showing a) the corresponding Voronoi mesh, b) a magnified triangle with link lines and stub lines labelled, and c) with edge lengths, $l_{\alpha}$, and link lengths, $\Delta_{\alpha}$, labelled for each port, $\alpha$.

means the timestep is constrained by

$$
\Delta t<\Delta_{\min } \sqrt{2 \mu \epsilon}
$$

where $\Delta_{\min }$ is the shortest link line length in the mesh.

To ensure that a stable algorithm is created and a physically reasonable timestep is chosen, Delaunay triangular meshes must be used, as explained in [7], where the link line lengths will never be negative. An example mesh with the corresponding link lines is shown in fig. 3a.

Once the transmission line network has been constructed, the response of the cell to a piecewise constant voltage signal (w.r.t. the division of time in time steps of length $\Delta t$ ) can be computed. After this initial setup, the resulting system conserves energy perfectly and its solution is known analytically i.e. the simulation during run-time can be guaranteed to have unconditional stability.

The computation is traditionally split into two parts: the scatter process where the reflection of voltages 
a)
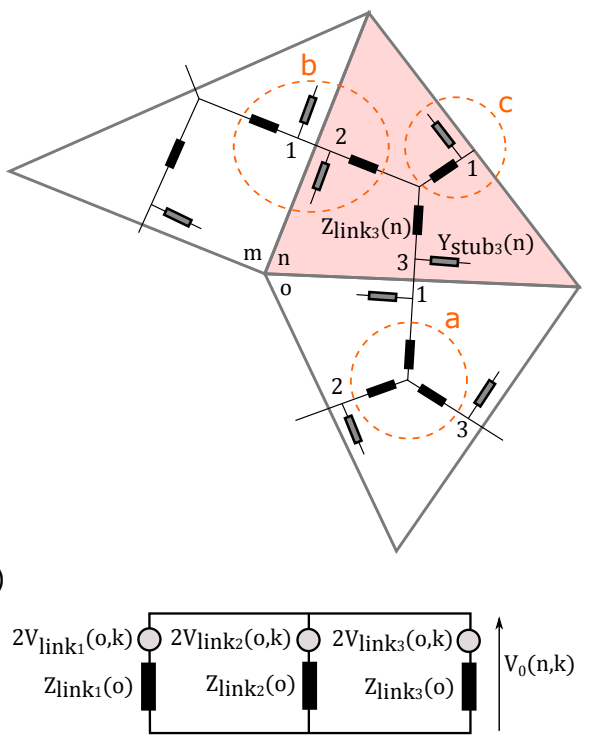

b)

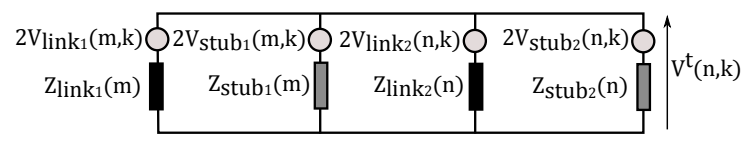

c)

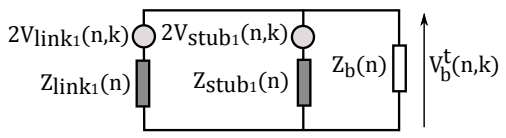

Fig. 4. The transmission lines inside UTLM triangles and the Thévenin equivalent circuit diagrams at timestep, $\mathrm{k}$, from a) the scatter process, b) connection between triangles, and c) connection at a boundary edge.

impinging on the triangle center is computed, and the connect process where the reflection of voltages impinging on the ports is computed. Both computations are based on the construction of a Thévenin equivalent, and on the splitting of the total voltage anywhere on a line into its subsequent incident and reflected voltages,

$$
V^{t}=V^{i}+V^{r}
$$

where superscript $t, i$, and $r$ denote the total, incident, and reflected values. The topology of the TLM circuits relevant to the scatter and connect steps is indicated in fig. 4.

\section{A. Scatter process}

The scatter process computes the voltages reflected from the triangle center using the voltages incident from the triangle edges and from the open end of the stub lines. The voltages incident on the end of the stub lines are simply reflected, whereas the voltages reflected by the triangle center can be found using the network in fig. $4 a$, which makes use of eq. (6).

$$
\begin{aligned}
& V_{\text {stub }}^{r}(n, k)=V_{\text {stub }}^{i}(n, k) \\
& V_{\text {link }_{\alpha}}^{r}(n, k)=V_{0}(n, k)-V_{\text {link }_{\alpha}}^{i}(n, k)
\end{aligned}
$$

for $\alpha=1,2,3$, where superscript $i$ and $r$ denote the incoming and reflected voltages respectively, and $V_{0}(n, k)$ is the total voltage in the centre of triangle $n$ at timestep $k$.

Investigation of the Thévenin equivalent circuit as shown in fig. $4 \mathrm{a}$ gives the total voltage at the centre of a triangle,

$$
V_{0}(n, k)=\frac{2 \sum_{\alpha=1}^{3} V_{\text {link }_{\alpha}}^{i}(n, k) Y_{\text {link }_{\alpha}}(n)}{\sum_{\alpha=1}^{3} Y_{\text {link }_{\alpha}}(n)} .
$$

\section{B. Connect process}

In the connect process, the voltages reflected at the inter-triangle ports are computed.

From the Thévenin equivalent in fig. $4 \mathrm{~b}$, the total voltage at the port between the triangles $m$ and $n$ is

$$
\begin{aligned}
& V^{t}(m, k)=V^{t}(n, k)=
\end{aligned}
$$

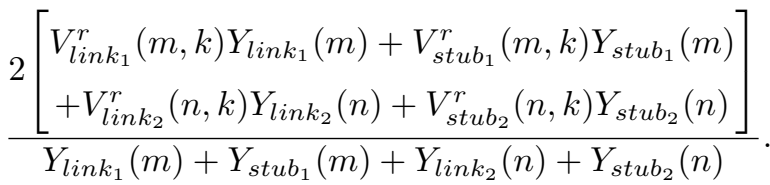

The incident link and stub voltages for the next timestep are

$$
\begin{aligned}
& V_{l i n k_{\alpha}}^{i}(n, k+1)=V^{t}(n, k)-V_{l i n k_{\alpha}}^{r}(n, k) \\
& V_{s t u b_{\alpha}}^{i}(n, k+1)=V^{t}(n, k)-V_{s t u b_{\alpha}}^{r}(n, k)
\end{aligned}
$$

for $\alpha=1,2,3$. These values are then used in the scatter process at the next timestep. 


\section{Connection at the boundaries}

To model the radiating behaviour of the fields at the TLM boundary of the problem space, the simplest approach is to terminate the mesh with a lumped impedance, the so called matched impedance, with value equal to the wave impedance of free space. This is indicated in the circuit of fig. 4c. In this situation the total voltage and current at the exterior edge becomes

$$
\begin{aligned}
V_{b}^{t}(n, k) & =\frac{I_{b}^{t}(n, k)}{Y_{\text {link }}(n)+Y_{\text {stub }}(n)+Y_{b}} \\
I_{b}^{t}(n, k) & =2 V_{\text {link }_{1}}^{r}(n, k) Y_{\text {link }_{1}}(n) \\
& +2 V_{\text {stub }}(n, k) Y_{\text {stub }}(n)
\end{aligned}
$$

where $Y_{b}$ is the boundary admittance.

Unfortunately, this is a crude approximation to a perfect radiating boundary condition that is inaccurate at non-smooth boundaries and for obliquely incident fields.

There are other methods to improve the boundary conditions, such as using Perfectly Matched Layers (PMLs) as described in [24], but these methods are more difficult to implement and currently cannot reach the level of performance that would make them comparable with Finite-Difference Time-Domain (FDTD) PML algorithms [25].

The following section describes a method for achieving a much more accurate $\mathrm{ABC}$.

\section{BEUT: COUPLING BEM AND UTLM}

The hybridization of BEM and UTLM is achieved by enforcing continuity of fields across the boundary interface.

As mentioned in the previous section, traditional matched boundaries for TLM consist of applying an impedance to terminate the transmission line as shown in fig. 5a. This does not take into account the interactions from all the other boundary edges. The Boundary Element/ Unstructured TLM or BEUT method here a)

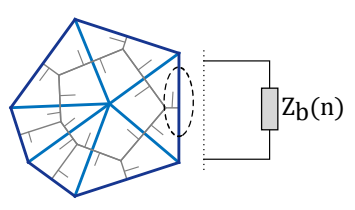

b)

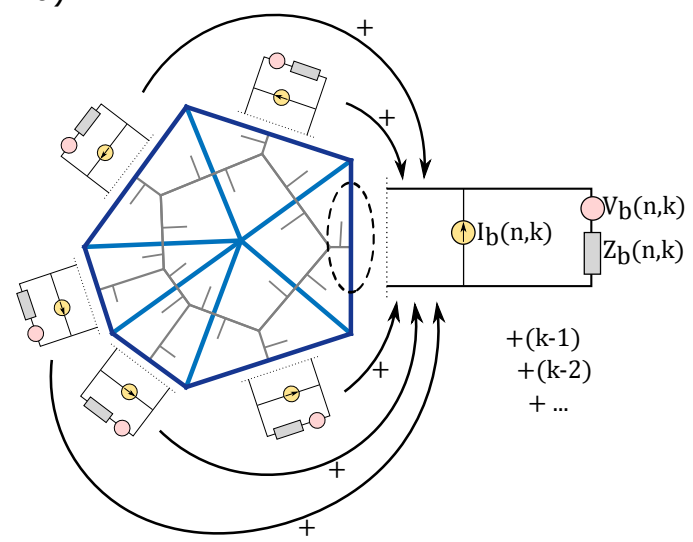

Fig. 5. The circuit representations of the boundary conditions used in a) classic TLM, and b) BEUT.

introduces the local boundary impedance with a nonlocal interaction matrix resulting from the representation theorem for the external domain. This operator takes into account contributions from voltages at all boundary edges, including those from multiple surfaces, and all previous timesteps. This can be thought of as replacing the exterior region with a multi-port TLM cell connecting all boundary edges at the current timestep and all boundary edges from all previous timesteps, as illustrated in fig. $5 b$.

Each link line and stub circuit model at the boundary can be reduced to a Thévenin and Norton equivalent circuit. The corresponding open port voltage and closed port current can be found as

$$
\begin{aligned}
V_{\text {open }} & =\frac{I_{\text {closed }}}{Y_{T L}} \\
I_{\text {closed }} & =2 V_{\text {link }}^{r} Y_{\text {link }}+2 V_{\text {stub }}^{r} Y_{\text {stub }}
\end{aligned}
$$

where the total transmission line admittance, $Y_{T L}=$ $Y_{\text {link }}+Y_{\text {stub }}$. This, in turn, is also the Thévenin-Norton equivalent of a single transmission line with admittance 
$Y_{T L}$ (and impedance $Z_{T L}=1 / Y_{T L}$ ) for which the following one-dimensional representation theorem holds:

$$
\begin{aligned}
& \left(\begin{array}{c}
V_{b} \\
I_{b}
\end{array}\right)= \\
& \left(\begin{array}{cc}
\frac{1}{2} & \frac{Z_{T L}}{2} \\
\frac{Y_{T L}}{2} & \frac{1}{2}
\end{array}\right)\left(\begin{array}{c}
V_{b} \\
I_{b}
\end{array}\right)+\left(\begin{array}{c}
\frac{V_{\text {open }}}{2} \\
-\frac{I_{\text {closed }}}{2}
\end{array}\right)
\end{aligned}
$$

where subscript $b$ denotes the boundary values, and the matrices $Y_{T L}$ and $Z_{T L}$ are diagonal. There is such a representation theorem for each edge on the boundary between the TLM and BEM governed domains. Arranging these in a large block diagonal system yields an interior representation theorem valid for the current time step.

Equations (14) and (1) provide the inner and outer representation theorems for the electric and magnetic traces. They can be combined into a single system for $\left(e_{z}, h_{t}\right)$, or their aliases $\left(V_{b}, I_{b}\right)$, that has a unique solution. Subtracting (2) from (14) yields the UTLM/BEM counterpart of the PMCHWT and boundary integral equation:

$$
\begin{aligned}
& \left(\begin{array}{c}
\mathrm{e}_{\mathrm{z}}^{\mathrm{i}} \\
\mathrm{h}_{\mathrm{t}}^{\mathrm{i}} l_{b}
\end{array}\right)+\frac{1}{2}\left(\begin{array}{c}
-V_{\text {open }} \\
I_{\text {closed }}
\end{array}\right)= \\
& \left(\begin{array}{cc}
-\mathbf{D} & \frac{Z_{T L}}{2}+\frac{\eta}{c} \mathbf{S} l_{b}{ }^{-1} \\
\frac{Y_{T L}}{2}+\frac{c}{\eta} \mathbf{N} l_{b} & \mathbf{D}^{\prime}
\end{array}\right)\left(\begin{array}{l}
V_{b} \\
I_{b}
\end{array}\right)
\end{aligned}
$$

The first term represents an exterior excitation and the second term represents the transmission line signals incident on the boundary.

The BEUT method requires the following steps to be taken for every timestep:

1) Perform the UTLM scatter process, then find $V_{\text {open }}$ and $I_{\text {closed }}$ using eq. (13).

2) Solve the coupling eq. (15) to obtain the boundary values.

3) Run the UTLM connect process using the updated boundary values.

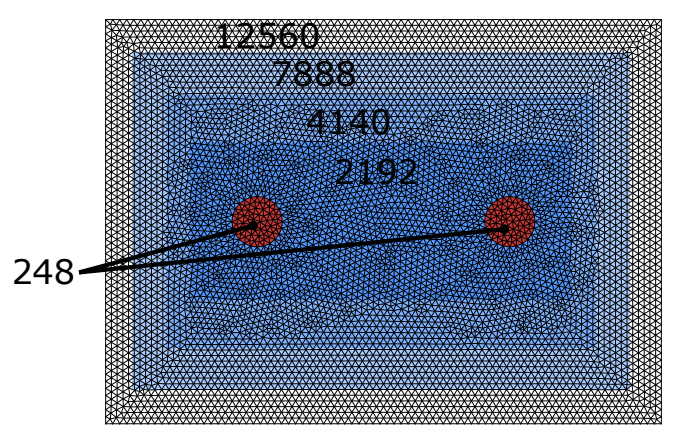

Fig. 6. Delaunay meshes demonstrating the different sized UTLM domains.

The voltages and currents that are the unknowns in the TLM description correspond to samples of the electric and magnetic field in the centers of interface edges (as shown in fig. 2a). This means that the choice of spatial testing functions and their normalisation for the BEM terms (as described in section II) allows for a natural mapping between TLM and BEM degrees of freedom.

An implementation using Matlab of the 2D UTLM, BEM, and BEUT methods, as described in this paper, can be found at https://github.com/dan-phd/BEUT. Kernels for matrix assembly that rely upon an optimised $\mathrm{C}++/$ openMP implementation can be found at https: //github.com/dan-phd/2DTDBEM.

\section{RESULTS}

Previous publications $[44,45]$ have proved the accuracy of the 2D BEUT method. Here we will demonstrate the suitability for problems that contain multiple smooth geometries containing inhomogeneous materials separated by significant quantities of free space. This ability is clearly an additional advantage of the BEUT method over traditional absorbing boundary conditions.

\section{A. Two Spatially Distinct Dielectric Cylinders}

Firstly, the interaction between two spatially distinct cylinders was investigated using BEUT, and the accuracy 


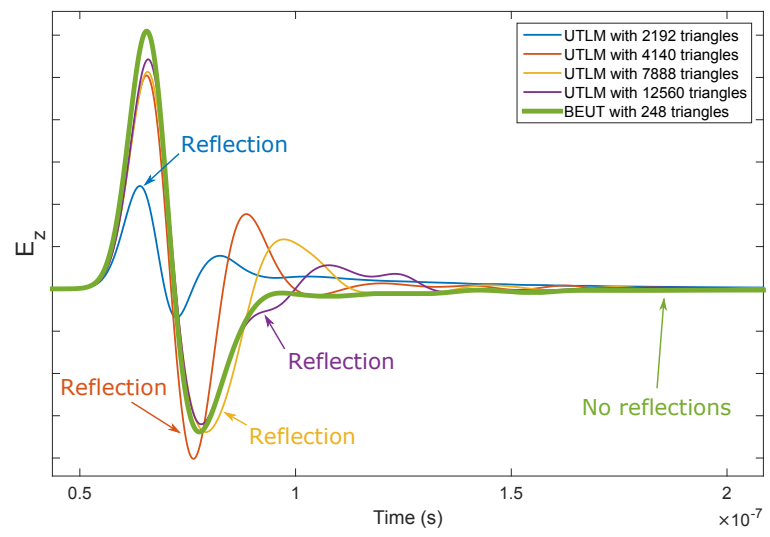

Fig. 7. Plots of time domain results when comparing BEUT with pure UTLM.

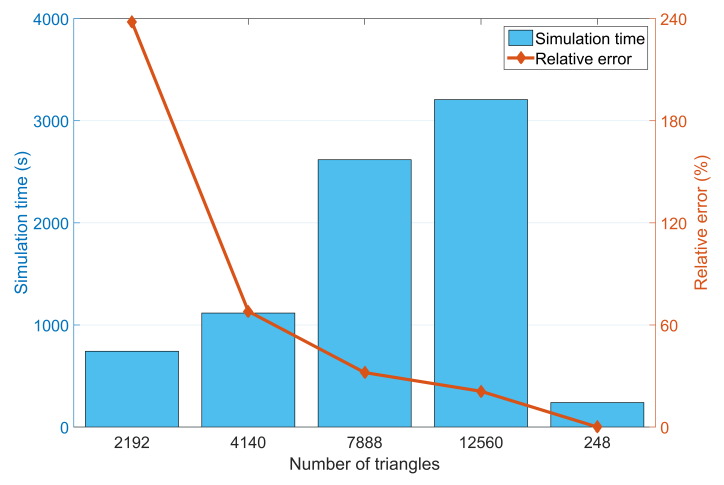

Fig. 8. Graph showing the speed and accuracy gains of BEUT over UTLM.

and speed was compared with a purely UTLM simulation. The diameter of the UTLM simulation domain was increased to reduce the effects of the artificial absorbing boundaries (as shown in Fig. 6), and re-compared with the results using BEUT to monitor the convergence.

Because BEUT only requires the objects to be meshed, there were just 248 triangles to model, making it much faster than using UTLM which requires the whole domain to be meshed. As predicted, the purely UTLM results showed evidence of non-physical reflections from the matched boundaries, which is illustrated in Fig. 7. The results from the BEUT method, however, contained no reflections and the perfectly radiating fields were

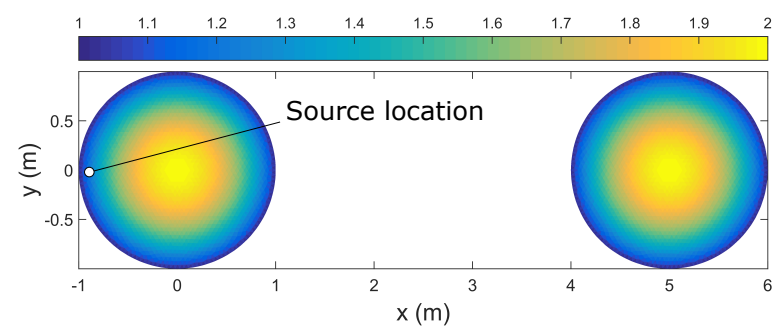

Fig. 9. Diagram showing the relative permittivity values across two Luneburg lenses, along with the point source location used in the test case.

observed. The graph in fig. 8 show that the UTLM results converge to the BEUT result as the mesh size increases. Fig. 8 also reveals the run-times that occurred during the test, which were much faster than UTLM.

\section{B. Two Spatially Distinct Luneburg Lens Antennas}

To demonstrate the practical application of the BEUT method, a point source signal transmitted over-the-air using two Luneburg lens antennas was modelled. A Luneburg lens is a non-uniform lens that transforms a spherical wave into a plane wave (and vice versa) $[46,47]$.

An ideal Luneburg lens is a radially symmetric sphere with a continuously varying relative permittivity ranging from 1 at the surface to 2 at the center,

$$
\epsilon_{r}(r)=2-\left(\frac{r}{a}\right)^{2}
$$

where $a$ is the radius, and $r$ is the distance from the center. Fig. 9 shows the relative permittivity variation and the location of the point source used in the test case.

To compute this problem using UTLM alone would be inefficient and inaccurate, as interference would occur from artificial reflections from the boundary of the simulation domain. On the other hand, BEM cannot be used to model transmission through the non-uniform Luneburg lens. Using BEUT enables accurate modelling of the lens, and accurate modelling of the transmitted 


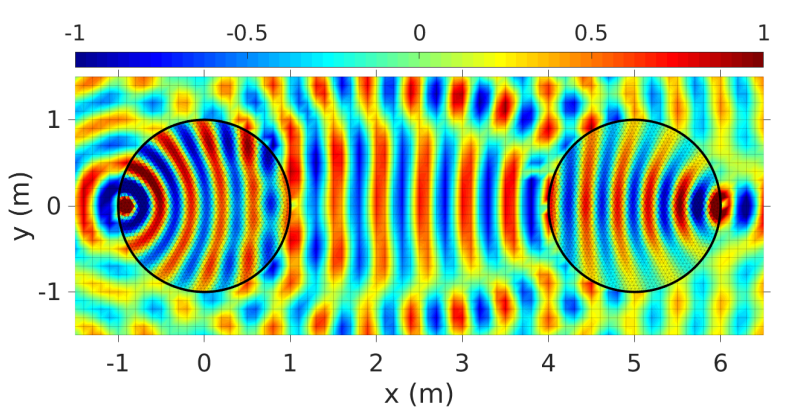

Fig. 10. 2D plot showing the total normalised electric field emanating between two Luneburg Lenses at a point in time.

waves through free space. Furthermore, only the lenses need to be meshed, so computational resources are conserved when compared to a fully meshed solver.

Fig. 10 displays the total electric field due to a point source positioned at the left lens. As can be seen, the incident wave produced by the point source is converted to a plane wave during transmission over the free space region, and then concentrated back to a point source at the right lens.

The fields inside the Luneburg lens antennas are modelled using UTLM, and the mesh shown in fig. 10 is the Delaunay triangulation used in this domain. All fields outside these regions, i.e. in free space, are modelled by BEM; the mesh shown in fig. 10 is an auxiliary mesh used for visualisation of the fields only.

\section{Dipole Antenna and Radome Interaction}

As a more applied example involving an irregular geometry, the effect of a radome positioned over a radiating antenna for aerospace communication is analysed here. A radome is a structural enclosure which protects an antenna from damage by the surrounding environment without effecting performance. A typical example would be the nose of an aircraft which protects the antenna beneath from aerodynamic stresses.

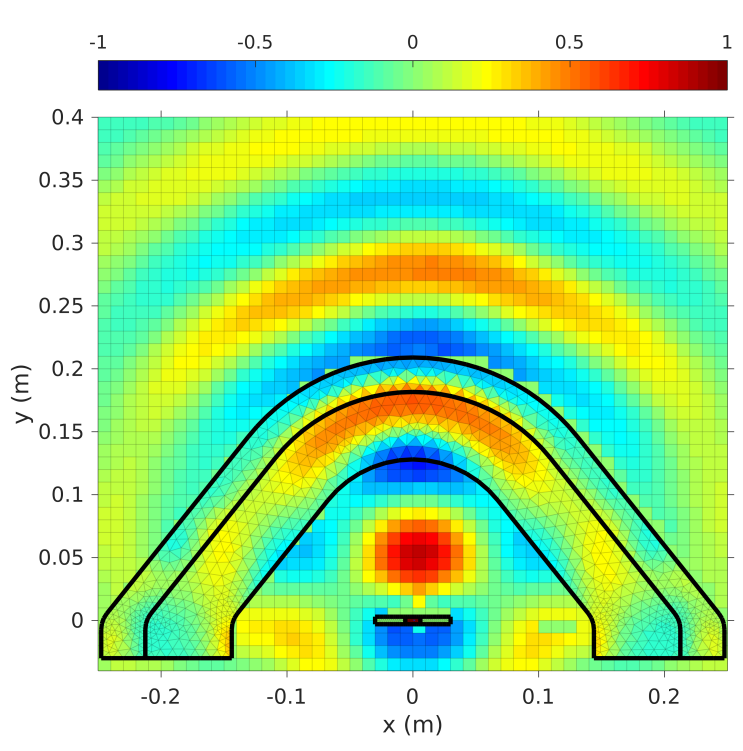

Fig. 11. 2D plot showing the total normalised electric field emanating between the $2.5 \mathrm{GHz}$ dipole antenna and radome at a point in time.

The radome design is tailored to the frequency range of the protected antenna, and depends on the materials used for its construction, the number of layers, and its shape. For this analysis, we will monitor a wireless LAN dipole antenna operating at $2.5 \mathrm{GHz}$, protected by a spherically blunted cone with a base inner radius of $1.2 \lambda$ and an inner height of $1 \lambda$. The radome is built with a $35 \mathrm{~mm}$ thick outer layer made of plastic polymer $\left(\varepsilon_{r}=4.8\right)$, and a $68.7 \mathrm{~mm}$ thick inner layer made of foam polyethylene $\left(\varepsilon_{r}=1.25\right)$.

The dipole antenna consists of two identical, perfectly conducting elements either side of a free space gap where the point source is located. The antenna length is $\lambda / 2$ and the structure is meshed very finely at roughly 80 edges per wavelength. The radome has a much more coarse mesh. It is common in the design of radomes to first compute the incident field radiated by the antenna, and then to model the radome using this incident field in a separate simulation, neglecting any mutual coupling. Using the BEUT method, the dome and the source 
geometry are modelled by a mesh with mesh size only dependent on the local geometry. This is in stark contrast to the structured TLM where the finest geometric detail determines the global mesh size. In addition, the empty space region in between dome and source is governed by the BEM, resulting in a technique that uses the bare minimum of degrees of freedom without jeopardising the solution's accuracy.

As can be seen in fig. 11, the fields inside the objects are modelled using UTLM with a triangular mesh. All fields outside these regions are modelled by BEM. In this case, the scattered field is found at points defined in a structured mesh with a $10 \mathrm{~mm}$ edge length.

To confirm the effectiveness of this particular radome, we can compare the far-field of the antenna with and without the radome at the design frequency of $2.5 \mathrm{GHz}$. The far-field array pattern can be obtained by measuring the electric field at a distance sufficiently far away from the source. It is directly available from the BEM boundary data and, because it is computed using the exact Green function of the propagation environment, its accuracy is not affected by dispersion error. This comparison can be seen in fig. 12, where the results are normalised w.r.t. the peak antenna response.

The results show that the forward signal is amplified when the radome is used at the design frequency. This is expected because each layer of the radome has a half wavelength thickness, which introduces a $360^{\circ}$ phase shift. Because of this phase shift, the reflections at each interface are superimposed causing an increase in the net transmission of waves.

Computing the far field using a purely UTLM based technique would require enlarging the simulation domain and even then the far field would be compromised by spurious reflections from the simulation domain boundary and accumulated dispersion errors.

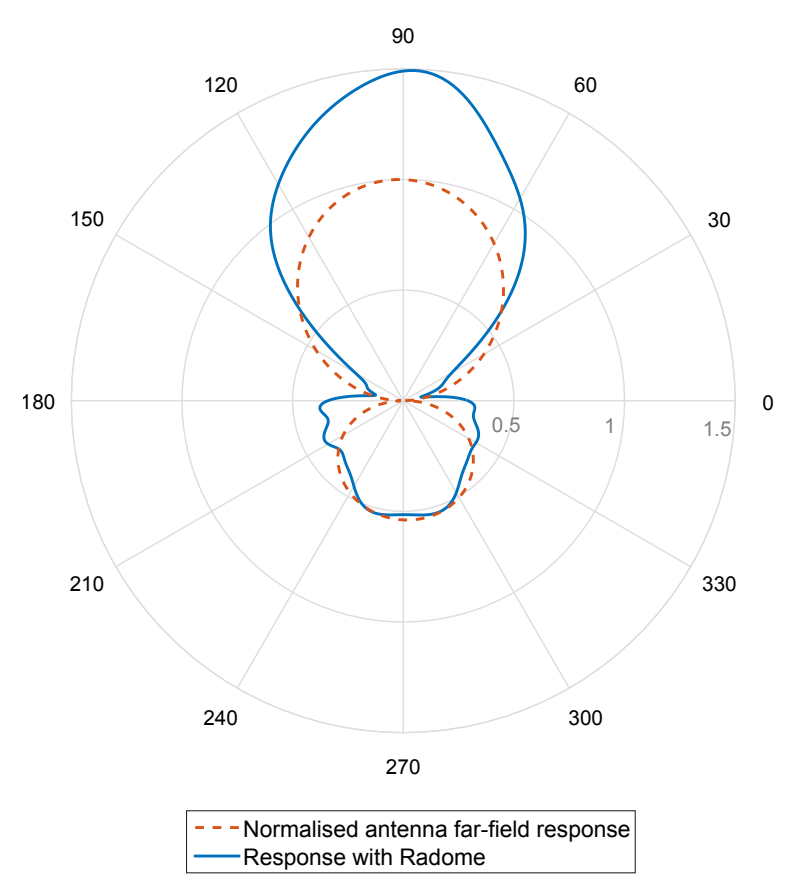

Fig. 12. Plot showing the total normalised electric far-field response from the dipole antenna at $2.5 \mathrm{GHz}$ with and without the radome.

\section{CONCLUSION}

This paper has presented the 2D BEUT method, a novel EM simulation technique which hybridizes the BEM and UTLM methods. The individual techniques were derived, and implementation guidelines were described.

The novel technique combines the power of UTLM (unconditional stability, ability to model inhomogeneous materials and smooth geometries) with the accuracy of BEM (perfectly radiating boundaries, resolvable fields anywhere in free space). To extend the technique to $3 \mathrm{D}$, the biggest challenge is the generalization of the map linking TLM and BEM degrees of freedoms, which may result in a discontinuous Galerkin scheme being used.

Simple test cases showed significant accuracy and speed gains compared to using pure UTLM. Scattering between two spatially distinct, non-uniform Luneburg 
lenses was shown using BEUT, and also the scattering between an antenna and a radome. Results obtained from the demonstrations matched that of expected and previously published results.

\section{ACKNOWLEDGEMENTS}

The authors gratefully acknowledge the financial support received from the UK Engineering and Physical Sciences Research Council (EPSRC) for the doctoral training grant that supported this work.

\section{REFERENCES}

[1] Y. Beghein, K. Cools, H. Bagci, D. De Zutter, A SpaceTime Mixed Galerkin Marching-on-in-Time Scheme for the Time-Domain Combined Field Integral Equation, IEEE Trans. Antennas Propag. 61 (3) (2013) 1228-1238. doi:10.1109/TAP.2012.2226553.

[2] D. Weile, G. Pisharody, A novel scheme for the solution of the time-domain integral equations of electromagnetics, Antennas ... 52 (1) (2004) 283-295.

[3] D. S. Weile, Accelerating convolution quadrature, in: 2015 Int. Conf. Electromagn. Adv. Appl., IEEE, Turin, 2015, pp. 341-344. doi:10.1109/ICEAA.2015.7297132.

[4] B. Shanker, A. Arif Ergin, K. Aygün, E. Michielssen, Analysis of transient electromagnetic scattering phenomena using a twolevel plane wave time-domain algorithm, IEEE Trans. Antennas Propag. 48 (4) (2000) 510-523. doi:10.1109/8.843664.

[5] M. Lu, E. Michielssen, B. Shanker, K. Yegin, Fast Time Domain Integral Equation Solvers for Analyzing Two-Dimensional Scattering Phenomena; Part I: Temporal Acceleration, Electromagnetics 24 (6) (2004) 425-449. doi:10.1080/02726340490467529.

[6] C. Christopoulos, The Transmission-Line Modeling Method TLM, The IEEE Series on Electromagnetic Wave Theory, Wiley, 1995.

[7] P. Sewell, J. Wykes, T. Benson, C. Christopoulos, D. Thomas, A. Vukovic, Transmission-line modeling using unstructured triangular meshes, IEEE Trans. Microw. Theory Tech. 52 (5) (2004) 1490-1497. doi:10.1109/TMTT.2004.827027.

[8] F. J. German, G. K. Gothard, L. S. Riggs, A. N. D. P. M. Goggans, THE CALCULATION OF RADAR CROSS-SECTION ( RCS ) USING THE TLM METHOD 2 (August 1989) (1990) 267-278.

[9] F. L. Teixeira, Time-domain finite-difference and finiteelement methods for Maxwell equations in complex media, IEEE Trans. Antennas Propag. 56 (8 I) (2008) 2150-2166. doi:10.1109/TAP.2008.926767.
[10] J. Paul, C. Christopoulos, D. Thomas, Generalized material models in TLM .I. Materials with frequency-dependent properties, IEEE Trans. Antennas Propag. 47 (10) (1999) 1528-1534. doi:10.1109/8.805895.

[11] J. Paul, C. Christopoulos, D. Thomas, Generalized material models in TLM .II. Materials with anisotropic properties, IEEE Trans. Antennas Propag. 47 (10) (1999) 1535-1542. doi:10.1109/8.805896.

[12] A. Taflove, S. C. Hagness, Computational ElectrodynamicsThe Finite Difference Time Domain Method, 3rd Edition, Artech House, 2005.

[13] S. D. Gedney, S. Member, J. A. Roden, Numerical Stability of NonorthogonalFDTD Methods, IEEE Trans. Antennas Propag. 48 (2) (2000) 231-239.

[14] D. B. Davidson, Computational Electromagnetics for RF and Microwave Engineering, 2nd Edition, Cambridge University Press, 2010.

[15] Lee Jin-Fa, Lee Robert, A. Cangellaris, Time-domain finiteelement methods, IEEE Trans. Antennas Propag. 45 (3) (1997) 430-442. doi:10.1109/8.558658.

[16] M. Lu, M. Lv, A. A. Ergin, B. Shanker, E. Michielssen, Multilevel plane wave time domain-based global boundary kernels for two-dimensional finite difference time domain simulations, Radio Sci. 39 (4). doi:10.1029/2003RS002928.

[17] B. Shanker, M. Lu, A. A. Ergin, E. Michielssen, Planewave time-domain accelerated radiation boundary kernels for FDTD analysis of 3-D electromagnetic phenomena, IEEE Trans. Antennas Propag. 53 (11) (2005) 3704-3716. doi:10.1109/TAP.2005.858590.

[18] D. Jiao, A. Ergin, B. Shanker, E. Michielssen, Jian-Ming Jin, A fast higher-order time-domain finite element-boundary integral method for 3-D electromagnetic scattering analysis, IEEE Trans. Antennas Propag. 50 (9) (2002) 1192-1202. doi:10.1109/TAP.2002.801375.

[19] A. E. Ylmaz, Z. Lou, E. Michielssen, J.-m. Jin, A Single-Boundary Implicit and FFT-Accelerated TimeDomain Finite Element-Boundary Integral Solver, IEEE Trans. Antennas Propag. 55 (5) (2007) 1382-1397. doi:10.1109/TAP.2007.895632.

[20] P. Sewell, Y. K. Choong, C. Christopoulos, An accurate thin-wire model for 3-D TLM simulations, IEEE Trans. Electromagn. Compat. 45 (2) (2003) 207-217. doi:10.1109/TEMC.2003.810812.

[21] S. Phang, A. Vukovic, H. Susanto, T. M. Benson, P. Sewell, Ultrafast optical switching using paritytime symmetric Bragg gratings, J. Opt. Soc. Am. B 30 (11) (2013) 2984. doi:10.1364/JOSAB.30.002984

[22] X. Meng, P. Sewell, S. Phang, A. Vukovic, T. M. Benson, Modeling Curved Carbon Fiber Composite (CFC) Structures in 
the Transmission-Line Modeling (TLM) Method, IEEE Trans. Electromagn. Compat. 57 (3) (2015) 384-390. arXiv:1502.01227, doi:10.1109/TEMC.2015.2400055.

[23] A. Elkalsh, A. Vukovic, P. D. Sewell, T. M. Benson, Electrothermal modelling for plasmonic structures in the TLM method, Opt. Quantum Electron. 48 (4) (2016) 263. doi:10.1007/s11082016-0542-x.

[24] J. Paul, C. Christopoulos, D. Thomas, Perfectly matched layer for transmission line modelling (TLM) method, Electron. Lett. 33 (9) (1997) 729. arXiv:arXiv:1011.1669v3, doi:10.1049/el:19970506.

[25] N. Pena, M. Ney, Absorbing-boundary conditions using perfectly matched-layer (PML) technique for three-dimensional TLM simulations, IEEE Trans. Microw. Theory Tech. 45 (10) (1997) 1749-1755. doi:10.1109/22.641722.

[26] J. L. Dubard, D. Pompei, Optimization of the PML Efficiency in 3-D TLM Method, IEEE Trans. Microw. Theory Tech. 48 (7 PART 1) (2000) 1081-1088. doi:10.1109/22.848490.

[27] J.-P. Berenger, Perfectly matched layer for the FDTD solution of wave-structure interaction problems, IEEE Trans. Antennas Propag. 44 (1) (1996) 110-117. doi:10.1109/8.477535.

[28] J. A. Roden, S. D. Gedney, Convolution PML (CPML): An efficient FDTD implementation of the CFS-PML for arbitrary media, Microw. Opt. Technol. Lett. 27 (5) (2000) 334-339. doi:10.1002/1098-2760(20001205)27:5;334::AIDMOP14¿3.0.CO;2-A.

[29] P. Jiang, E. Michielssen, Multilevel Plane Wave Time DomainEnhanced MOT Solver for Analyzing Electromagnetic Scattering from Objects Residing in Lossy Media, in: 2005 IEEE Antennas Propag. Soc. Int. Symp., Vol. 3B, IEEE, 2005, pp. 447-450. doi:10.1109/APS.2005.1552540.

[30] Pei-Lin Jiang, E. Michielssen, Multilevel PWTD-Enhanced CFIE Solver for Analyzing EM Scattering from PEC Objects Residing in Lossy Media, in: 2006 IEEE Antennas Propag. Soc. Int. Symp., Vol. 11270, IEEE, 2006, pp. 2967-2970. doi:10.1109/APS.2006.1711230.

[31] A. E. Yilmaz, D. S. Weile, B. Shanker, J. M. Jin, E. Michielssen, Fast analysis of transient scattering in lossy media, IEEE Antennas Wirel. Propag. Lett. 1 (1) (2002) 14-17. doi:10.1109/LAWP.2002.802577.

[32] H. Baci, A. E. Yilmaz, V. Lomakin, E. Michielssen, Fast solution of mixed-potential time-domain integral equations for half-space environments, IEEE Trans. Geosci. Remote Sens. 43 (2) (2005) 269-279. doi:10.1109/TGRS.2004.841489.

[33] J. Paul, C. Christopoulos, D. W. P. Thomas, Generalized material models in TLM .III. Materials with nonlinear properties, IEEE Trans. Antennas Propag. 50 (7) (2002) 997-1004. doi:Doi 10.1109/Tap.2002.800733.

[34] L. Pierantoni, S. Lindenmeier, P. Russer, A Combination of Integral Equation Method and FD/TLM Method for Efficient
Solution of EMC Problems, 27th Eur. Microw. Conf. 19972 (1997) 937-942. doi:10.1109/EUMA.1997.337916.

[35] S. Lindenmeier, L. Pierantoni, P. Russer, Adapted radiating boundaries (ARB) for efficient time domain simulation of electromagnetic interferences, 1998 IEEE MTT-S Int. Microw. Symp. Dig. (Cat. No.98CH36192) 2 (1998) 465-468. doi:10.1109/MWSYM.1998.705033.

[36] S. Lindenmeier, L. Pierantoni, P. Russer, Hybrid Space Discretizing - Integral Equation Methods for Numerical Modeling of Transient Interference, IEEE Trans. Electromagn. Compat. 41 (4) (1999) 425-430. doi:10.1109/15.809843.

[37] S. Lindenmeier, L. Pierantoni, P. Russer, Numerical modelling of transient radiated interferences in time domain by the hybrid ARB method, Int. J. Numer. Model. Electron. Networks, Devices Fields 12 (4) (1999) 295-309. doi:10.1002/(SICI)10991204(199907/08)12:4¡295::AID-JNM331;3.0.CO;2-9.

[38] S. Lindenmeier, C. Christopoulos, P. Russer, Methods for the modeling of thin wire structures with the TLM method, 2000 IEEE MTT-S Int. Microw. Symp. Dig. (Cat. No.00CH37017) 1 (2000) 387-390.

[39] M. Zedler, G. V. Eleftheriades, Anisotropic transmissionline metamaterials for 2-d transformation optics applications, Proc. IEEE 99 (10) (2011) 1634-1645. doi:10.1109/JPROC.2011.2114310.

[40] M. Naser-Moghadasi, M. Bahadorzadeh, R. Sadeghzadeh, Implementation of a Novel TLM-MOM Hybrid Method for the Analysis of Interference in Antennas, 2008 3rd Int. Conf. Inf. Commun. Technol. From Theory to Appl. (2008) 14doi:10.1109/ICTTA.2008.4530258.

[41] Y. Beghein, K. Cools, F. P. Andriulli, D. De Zutter, E. Michielssen, A calderon multiplicative preconditioner for the PMCHWT equation for scattering by chiral objects, IEEE Trans. Antennas Propag. 60 (2012) 4239-4248. doi:10.1109/TAP.2012.2207061.

[42] G. C. Hsiao, W. Wendland, Boundary Integral Equations, in: Theor. Numer. Anal., Springer, Berlin, 2008. doi:10.1007/978-14419-0458-4.

[43] K. Cools, Mortar boundary elements for the EFIE applied to the analysis of scattering by PEC junctions, cccc2012 Asia-Pacific Symp. Electromagn. Compat. APEMC 2012 - Proc. (2012) 165168doi:10.1109/APEMC.2012.6237847.

[44] D. Simmons, K. Cools, P. Sewell, Coupling of unstructured TLM and BEM for accurate 2D electromagnetic simulation, in: 2015 Int. Conf. Electromagn. Adv. Appl., IEEE, Torino, 2015, pp. 1076-1079. doi:10.1109/ICEAA.2015.7297281.

[45] D. Simmons, K. Cools, P. Sewell, Modelling antennas in outer space using the boundary element unstructured transmission-line (BEUT) method, in: 36th ESA Antenna Work. Antennas RF Syst. Sp. Sci., Noordwijk, 2015. 
[46] H. Mosallaei, Y. Rahmat-Samii, Nonuniform Luneburg and twoshell lens antennas: radiation characteristics and design optimization, IEEE Trans. Antennas Propag. 49 (1) (2001) 60-69. doi:10.1109/8.910531.

[47] M. Bosiljevac, M. Casaletti, F. Caminita, Z. Sipus, S. MacI, Non-uniform metasurface luneburg lens antenna design, IEEE Trans. Antennas Propag. 60 (9) (2012) 4065-4073. doi:10.1109/TAP.2012.2207047. 